\title{
Anacardic Acids and Ferric Ion Chelation
}

\author{
Kazuo Tsujimoto ${ }^{\mathrm{a}, *}$, Akio Hayashi ${ }^{\mathrm{a}}$, Tae Joung $\mathrm{Ha}^{\mathrm{b}}$, and Isao Kubo ${ }^{\mathrm{b}}$ \\ a School of Material Sciences, Japan Advanced Institute of Science and Technology, Nomi, \\ Ishikawa 923-1292, Japan. Fax: +81-761-51-1665. E-mail: tujimoto@jaist.ac.jp \\ b Department of Environmental Science, Policy and Management, University of California, \\ Berkeley, California 94720-3114, USA \\ * Author for correspondence and reprint requests \\ Z. Naturforsch. 62c, 710-716 (2007); received April 5/May 2, 2007 \\ 6-Pentadeca(e)nylsalicylic acids isolated from the cashew Anacardium occidentale L. (An- \\ acardiaceae), commonly known as anacardic acids, inhibited the linoleic acid peroxidation \\ catalyzed by soybean lipoxygenase-1 (EC 1.13.11.12, type 1) competitively without prooxi- \\ dant effects. Their parent compound, salicylic acid, did not have this inhibitory activity up to \\ $800 \mu \mathrm{M}$, indicating that the pentadeca(e)nyl group is an essential element to elicit the activity. \\ The inhibition is attributed to its ability to chelate iron in the enzyme. Thus, anacardic acids \\ chelate iron in the active site of the enzyme and then the hydrophobic tail portion slowly \\ begins to interact with the hydrophobic domain close to the active site. Formation of the \\ anacardic acids-ferric ion complex was detected in the ratio of $2: 1$ as the base peak in the \\ negative ion electrospray ionization mass spectrometry. Hence, anacardic acids inhibit both \\ $\mathrm{E}_{\mathrm{ox}}$ and $\mathrm{E}_{\mathrm{red}}$ forms.
}

Key words: Anacardic Acids, Lipoxygenase, Iron, Deprotonated Molecule

\section{Introduction}

Lipoxygenases (EC 1.13.11.12) are a family of non-heme iron-containing dioxygenases widely distributed in both the animal and the plant kingdoms. They catalyze the regio- and stereospecific oxygenation of polyunsaturated fatty acids, containing $(1 Z, 4 Z)$-pentadiene moieties, into the corresponding conjugated hydroperoxides. In plants, the main substrates are linoleic $\left(\mathrm{C}_{18: 2}\right)$ and linolenic $\left(\mathrm{C}_{18: 3}\right)$ acids, and the primary products of lipoxygeneses, $9 S$ and $13 S$ fatty acid hydroperoxides, are proposed to have regulatory roles in plant and animal metabolism (Gardner, 1995). Due to their free radical nature, fatty acid hydroperoxides can be quite active by themselves and are capable of producing membrane damage and promoting cell death (Gardner, 1991). Lipoxygenases are suggested to be involved in the early event of atherosclerosis by inducing plasma low-density lipoprotein (LDL) oxidation (Cornicelli and Trivedi, 1999; Kris-Etherton and Keen, 2002). On the other hand, it is well known that lipid peroxidation is one of the major factors in deterioration during the storage and processing of foods, because it can lead to the development of unpleasant rancid or off-flavors as well as potentially toxic end products (Grechkin, 1998). Hence, lipoxygenase inhibitors should have broad applications (Richard-Forget et al., 1995).

Three 6-pentadeca(e)nylsalicylic acids [pentadeca(e)nyl is a collective term of side chains of anacardic acids] commonly known as anacardic acids and isolated from the cashew Anacardium occidentale L. (Anacardiaceae); 6[8(Z),11(Z),14pentadecatrienyl]salicylic acid $(\mathbf{1}), 6[8(Z), 11(Z)$ pentadecadienyl]salicylic acid (2), and 6[8(Z)-pentadecenyl]salicylic acid (3) (reviewed by Tyman, 1979); were reported to inhibit the linoleic acid peroxidation catalyzed by soybean lipoxygenase- 1 (Nagabhushana et al., 1995). Recently, 3 was further studied and found to have the same inhibition without prooxidant effects (Ha and Kubo, 2005). The aim of this paper is to describe the chelation ability of these acids and pentadeca(e)nyl side chain effects.

\section{Materials and Methods}

\section{Chemicals}

Anacardic acids $\left(\mathrm{C}_{15: 0}, \mathrm{C}_{15: 1}, \mathrm{C}_{15: 2}, \mathrm{C}_{15: 3}\right)$ and the corresponding methyl esters and acetates were available from our previous work (Kubo et al., 1986, 1993, 1994; Yamagiwa et al., 1987). Soybean lipoxygenase-1 (EC 1.13.11.12, type 1), dimethyl sulfoxide (DMSO), salicylic acid, Tween-20 and 
linoleic acid were purchased from Sigma Chemical Co. (St. Louis, MO). 13-Hydroperoxyoctadecadienoic acid (13-HPOD: $\lambda_{\max }=234 \mathrm{~nm}, \varepsilon=$ $25 \mathrm{~mm}^{-1} \mathrm{~cm}^{-1}$ ) was prepared enzymatically by a described procedure (Gibian and Galaway, 1976) and stored in ethanol at $-18^{\circ} \mathrm{C}$.

\section{ESI mass spectrometry}

Electrospray ionization (ESI) mass spectra were obtained on a Finnigan LCQ-Deca XP (Thermo Fisher Scientific, Inc, Waltham, MA) mass spectrometer. The mass spectrometer was equipped with an ESI needle and the ion spray voltage was set at $3 \mathrm{kV}$ with nitrogen as the sheath gas. Mass spectra were acquired at the mass range $\mathrm{m} / \mathrm{z}, 50-$ 2000 in the negative ion mode to detect deprotonated molecules. The complex solution was directly infused into the mass spectrometer at a flow rate of $3 \mu \mathrm{L} / \mathrm{min}$. For all the ESI measurements of the hydrophobic complex ion, a non-aqueous solution was prepared: 2-propanol/hexane/ethyl acetate, $2: 1: 1$, with $0.05 \%$ acetic acid. A hexane solution of anacardic acid and an ethyl acetate solution of ferric acetylacetonate in a ratio of $1: 2$, respectively, were added to the solution and adjusted to a final concentration of $10 \mathrm{pmol} / \mu \mathrm{L}$.

\section{Inhibition experiments on lipoxygenase-1}

The assay was conducted in triplicate of separate experiments. The experiments were performed by measurement of the initial rate of soybean lipoxygenase- 1 with a Spectra MAX plus spectrophotometer (Molecular Device, Sunnyvale, $\mathrm{CA})$ at $25^{\circ} \mathrm{C}$. The enzyme assay was performed as previously reported (Rickert and Klinman, 1999) with slight modification. In general, the reaction mixture consisted of $2.97 \mathrm{~mL}$ of $0.1 \mathrm{~m}$ sodium borate buffer ( $\mathrm{pH} 9.0$ ), $15 \mu \mathrm{L}$ of $3 \mathrm{~mm}$ stock solution of linoleic acid and $5 \mu \mathrm{L}$ of an ethanolic inhibitor solution. Then, $10 \mu \mathrm{L}$ of $0.1 \mathrm{~m}$ sodium borate buffer solution ( $\mathrm{pH} 9.0)$ of lipoxygenase $(0.52 \mu \mathrm{M})$ were added. The resultant solution was mixed well and the linear increase of absorbance at $234 \mathrm{~nm}$ for $5 \mathrm{~min}$, which expresses the formation of conjugated diene hydroperoxide (13-HPOD, $\varepsilon=$ $25000 \mathrm{M}^{-1} \mathrm{~cm}^{-1}$ ), was measured continuously. Lag period shown on lipoxygenase reaction (Ruddat $e t$ al., 2003) was excluded for the determination of initial rates. The stock solution of linoleic acid was prepared with Tween-20 and sodium borate buffer at $\mathrm{pH}$ 9.0, and then total Tween-20 content in the final assay was adjusted below $0.01 \%$. The data analysis was performed by using Sigma Plot 2000 (SPSS Inc, Chicago, IL). The $\mathrm{IC}_{50}$ values were obtained by fitting experimental data to the logistic curve by the equation (Copeland, 2000)

$$
\text { activity }(\%)=100\left[1 /\left(1+\left([\mathrm{I}] / \mathrm{IC}_{50}\right)\right)\right] \text {, }
$$

where $[\mathrm{I}]$ is the concentration of the inhibitor.

\section{Results}

For simplicity, the three 6-pentadeca(e)nylsalicylic acids are referred to anacardic acid $\mathrm{C}_{15: 3}(\mathbf{1})$, anacardic acid $\mathrm{C}_{15: 2}(\mathbf{2})$, and anacardic acid $\mathrm{C}_{15: 1}$ (3), respectively (see Fig. 1 for structures). In addition, since anacardic acids are the derivatives of salicylic acid (4) with a non-isoprenoid pentadeca(e)nyl side chain at the C-6 position, the activity was compared with that of salicylic acid.

Deleterious free radical-mediated oxidations occur in aerobic organisms as a result of normal oxygen metabolism. The human body produces free radicals during the course of its normal metabo-

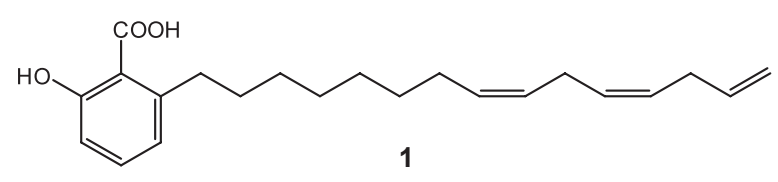<smiles>CCC/C=C/C/C=C/CCCCCCCc1cccc(O)c1C(=O)O</smiles><smiles>CCCCCC/C=C/CCCCCCCc1cccc(O)c1C(=O)O</smiles><smiles>O=C(O)c1ccccc1O</smiles>

Fig. 1. Chemical structures of anacardic acids and related compounds: $\quad 6[8(Z), 11(Z), 14$-pentadecatrienyl]salicylic acid (1, anacardic acid $\left.\mathrm{C}_{15: 3}\right), 6[8(Z), 11(Z)$-pentadecadienyl]salicylic acid $\left(2\right.$, anacardic acid $\left.C_{15: 2}\right), 6[8(Z)$-pentadecenyl]salicylic acid (3, anacardic acid $\left.\mathrm{C}_{15: 1}\right)$, and salicylic acid (4). 
lism. Free radicals are even required for several normal biochemical processes. For example, the phagocyte cells involved in the body's natural immune defenses generate free radicals in the process of destroying microbial pathogens. If free radicals are produced during the normal cellular metabolism in sufficient amounts to overcome the normally efficient protective mechanisms, metabolic and cellular disturbances will occur in a variety of ways. Evidence is accumulating that several cell types other than phagocytes also produce extracellular free radicals in vivo. For example, lipids are oxidized by lipoxygenases and cyclooxygenases, which generate peroxide intermediates. More specifically, lipoxygenases catalyze the oxygenation of polyenoic fatty acids containing a $(1 Z, 4 Z)$ pentadiene system, such as linoleic acid and arachidonic acid, to their 1-hydroperoxy-(2E,4Z)pentadiene product. In this connection, lipoxygenases are of importance, since they may generate peroxides in human low-density lipoproteins in vivo and facilitate the development of arteriosclerosis, a process in which lipid peroxidation appears to be intimately involved (Ruddat et al., 2003). Anacardic acid $\mathrm{C}_{15: 1}$ inhibited the linoleic acid peroxidation catalyzed by soybean lipoxygenase- 1 without prooxidant effects. Thus, $30 \mu \mathrm{m}(10.4 \mu \mathrm{g} /$
$\mathrm{mL}$ ) of anacardic acid $\mathrm{C}_{15: 1}$ inhibited $80 \%$ of the linoleic acid peroxidation and the $\mathrm{IC}_{50}$ value was established as $6.8 \mu \mathrm{M}(2.35 \mu \mathrm{g} / \mathrm{mL})$.

Lipoxygenase is a non-heme iron-containing enzyme and lipoxygenase inhibitors are known to act mainly in two different ways: by chelating the iron of the active site of enzyme (Clapp et al., 1985; Nelson, 1988) and/or by reducing the ferric form of the enzyme to an inactive ferrous form (Kemal et al., 1987; Mansuy et al., 1988). Anacardic acids do not have reducing ability but, because of the salicylic acid moiety with a pentadeca(e)nyl side chain, it therefore appears that they can be expected to chelate iron in lipoxygenase. The UV absorption bands of anacardic acids should shift by adding excess ferrous ions and characteristic bathochromic shifts were observed by adding excess ferrous ions, similar to the shifts previously described by adding excess bicupric ions (Kubo et al., 2000). However, the expected shift was not observed in the absorption spectrum of the complex of anacardic acids and soybean lipoxygenase1 because the absorption overlapped with that of the enzyme itself.

On the other hand, anacardic acids were previously described to demonstrate high selectivity toward transition metal ions such as iron and copper
Fig. 2. Negative ESI mass spectrum of the complex; $1: 1$ mixture of anacardic acids $\left(\mathrm{C}_{15: 3}\right.$ and $\left.\mathrm{C}_{15: 1}\right)$ forming the complex with ferric acetylacetonate.

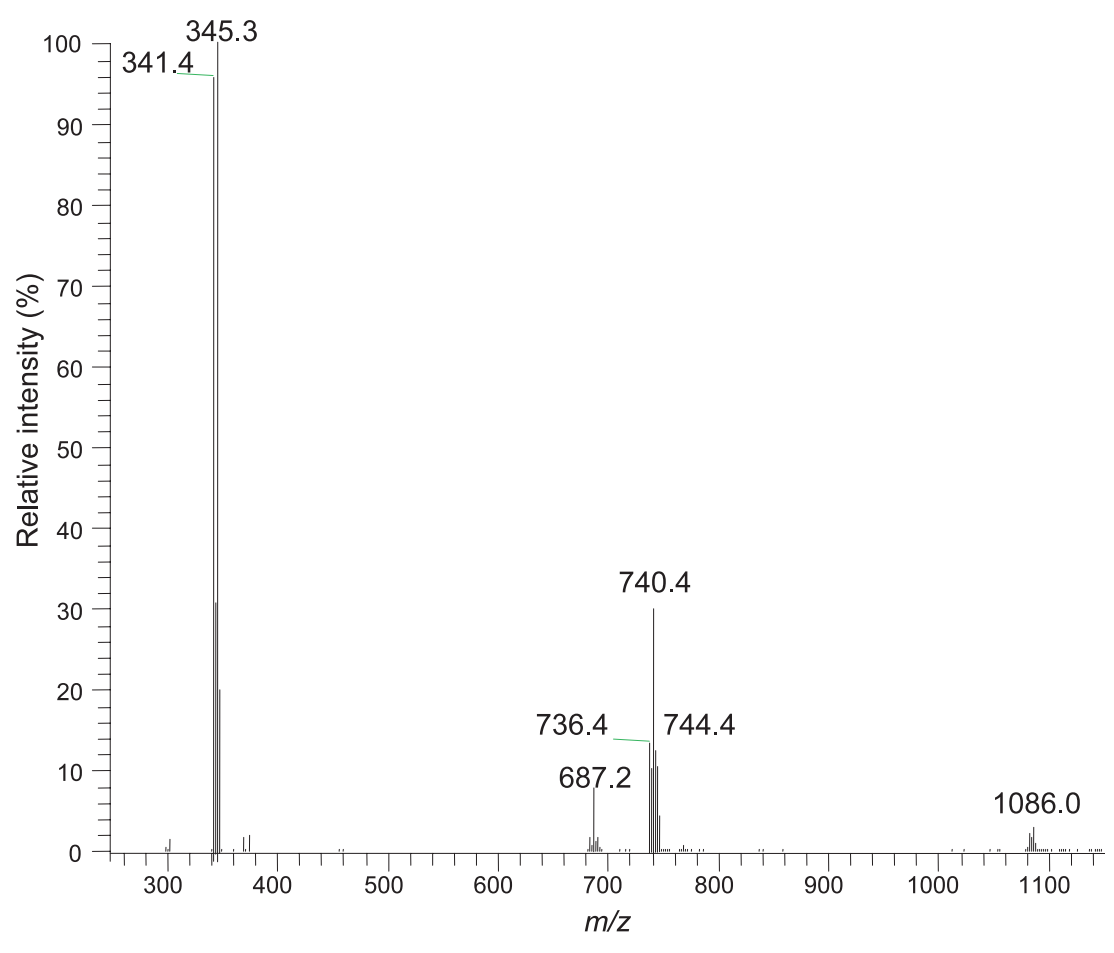


based on the absorption intensities (Nagabhushana et al., 1995). Although the divalent ferrous ion was capable of forming the complex, the complexation activity of the trivalent ferric ion still remained unsolved. Thus native soybean lipoxygenase- 1 contains a non-heme ferrous ion that must be oxidized to yield the catalytically active ferric enzyme (Kemal et al., 1987). The primary dioxygenation product in plants is $13(S)$-hydroperoxy9Z,11E-octadecadienoic acid (13-HPOD) (5) (Grechkin, 1998), and a catalytic amount of 13HPOD is usually added as cofactor. In order to obtain more accurate information about the metal-complex composition electrospray ionization mass spectrometry was effectively employed. In this way, formation of the anacardic acid $\mathrm{C}_{15: 3^{-}}$ ferric ion complex was successfully detected by negative ion electrospray ionization mass spectrometry [(-)-ESI-MS] (Hayashi et al., 2007). The spectrum showed the base peak at $\mathrm{m} / z, 736.4$ related to a 2:1 complex of anacardic acid $\mathrm{C}_{15: 3}$-ferric ion, as shown in Fig. 2. Furthermore, a weak peak (less than $10 \%$ ) of the anacardic acid $\mathrm{C}_{15: 3^{-}}$ ferric ion complex in the ratio of $3: 1$ was observed at $\mathrm{m} / \mathrm{z}, 1079.8$. Hence, the complexation preferred ratio of $2: 1$ opposite to a ratio of $3: 1$. The similar phenomena were also observed in the case of salicylic acid, but salicylic acid did not inhibit the linolenic acid peroxidation catalyzed by soybean lipoxygenase- 1 up to $800 \mu \mathrm{m}$. The results observed indicate that anacardic acids chelate both ferric and ferrous ions and therefore inhibit both $E_{o x}$ and $E_{\text {red }}$ forms (Fig. 3). However, it still lacks direct evidence that anacardic acids chelate iron in lipoxygenase.

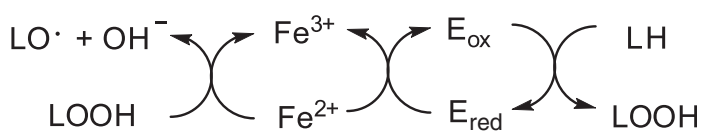

Fig. 3. Commercial lipoxygenase contains a non-heme ferrous ion $\left(\mathrm{E}_{\mathrm{red}}\right)$ that must be oxidized to yield the catalytically active ferric enzyme $\left(\mathrm{E}_{\mathrm{ox}}\right)$ and therefore a catalytic amount of LOOH (13-HPOD) is usually added as a cofactor to LH (linoleic acid, a substrate).

The iron chelation mechanism can be indirectly supported by the observation that neither the methyl ester nor the acetate of anacardic acid $\mathrm{C}_{15: 3}$ inhibited the oxidation up to $200 \mu \mathrm{M}$, demonstrating that both an alk(en)yl side chain tail as well as the salicylic acid head moiety are an essential combination to elicit the inhibitory activity similar to the previous report for the tyrosinase inhibitory activity (Nomura and Fujihara, 1994) and xanthine oxidase inhibitory activity (Masuoka and Kubo, 2004). On the other hand, EDTA (ethylene diaminetetraacetic acid), a well-known chelator, did not inhibit the linolenic acid peroxidation catalyzed by soybean lipoxygenase- 1 thereby indicating that the chelation ability alone is not enough to inhibit the activity of the enzyme.

In addition, transition metals are well known as powerful promoters of free radical damage in both the human body (Halliwell and Gutteridge, 1989; Henle and Linn, 1997) and foods (Aruoma and Halliwell, 1991). The ability to chelate transition metal ions should be a considerable advantage of anacardic acids as antioxidants. Anacardic acids may suppress the superoxide-driven Fenton reaction, which is currently believed to be the most important route to active oxygen species. For example, anacardic acids may prevent cell damage induced by $\mathrm{H}_{2} \mathrm{O}_{2}$, since this can be converted to the more reactive oxygen species, hydroxyl radicals in the presence of these metal ions (Lodovici et al., 2001). Thus, metal chelation may play an important role in determining the antioxidant activity (Arora et al., 1998). The chelation ability, rendering the metal ions inactive to participate in free radical generating reactions, should be of considerable advantage as antioxidants.

Anacardic acids are salicylic acid derivatives with a pentadeca(e)nyl side chain. They are amphipathic molecules and therefore their hydrophobic properties dominate the properties of the molecules. Thus, anacardic acids are expected to act as surface active agents (surfactants). The low conformational stability of native proteins makes them susceptible to denaturation by altering the balance of the weak non-bonding forces that maintain the native conformation. However, since native proteins form a sort of intramolecular micelle in which their non-polar portions are largely out of contact with the water-based test solution, the hydrophobic pentadeca(e)nyl side chain at C-6 may act as an anchor in the low dielectric interior of proteins. On the other hand the hydrophilic head portion of anacardic acids first binds to this portion of the enzyme molecule after which the hydrophobic tail portion slowly begins to interact with the $C$-terminal domain where the iron is located (Prigge et al., 1997). Since salicylic acid lacks this anchor, it cannot disrupt the hydrophobic domain. 
Anacardic acids are also known to inhibit tyrosinase (EC 1.14.18.1) competitively without prooxidant effects (Kubo et al., 1994). Tyrosinase is a copper-containing enzyme, and chelators such as kojic acid and tropolone are known to inhibit this mixed-function oxidase by chelating copper in the active site of the enzyme (Sánchez-Ferrer et al., 1995). Molecular observation of the metal complexes by mass spectrometry (MS) enables to estimate the composition of the complex. In the negative spectrum, the anacardic acid $\mathrm{C}_{15: 3}$-bicupric complex in a ratio of $2: 1$ was detected at $m / z, 744.4$ as the base peak. This peak underwent to produce several fragments including a weak peak at $\mathrm{m} / \mathrm{z}$. 403.2 of an anacardic acid $\mathrm{C}_{15: 3}$-bicupric complex in a ratio of $1: 1$. Even if the metal concentration was high, the latter peak was detected in a portion less than $3 \%$, indicating that the complexation preferred the ratio of $2: 1$ opposite to the ratio of $1: 1$. These phenomena were also observed in the case of salicylic acid (Hayashi et al., 2007). However, EDTA, a well known chelator, did not inhibit the tyrosinase-catalyzed oxidation of L-DOPA, indicating that the chelation ability alone is not enough to inhibit the enzyme. Similar to the aforementioned points, for the case of lipoxygenase, it proves no noticeable direct evidence that anacardic acids chelate copper in tyrosinase. The binuclear copper active site in the enzyme has a much more crowded environment and makes the salicylic acid moiety in anacardic acids more difficult to approach.

\section{Discussion}

Anacardic acids are known to inhibit various enzymes such as urease (Kubo et al., 1999), glycerol3-phosphate dehydrogenase (Irie et al., 1996), lipoxygenases (Shobha et al., 1994), $\beta$-lactamase (Coates et al., 1994; Hird and Milner, 1994), aldose reductase (Toyomizu et al., 1993), prostaglandin endoperoxide synthase (Grazzini et al., 1991), and prostaglandin synthase (Kubo et al., 1987; Bhattacharya et al., 1987). Since salicylic acid has little or no effects on these enzymes, the hydrophobic pentadeca(e)nyl side chain in anacardic acids is undoubtedly associated with the enzyme inhibitory activity. In addition, the number of double bonds in the side chain is not directly associated with the enzyme inhibitory activity (Shobha et al., 1994), suggesting that interaction of the double bond with a specific amino acid residue in the enzymes is unlikely. Anacardic acids are amphipathic molecules, so that their hydrophobic properties dominate the properties of the molecule. The precise explanation still remains unclear, but it seems that these enzymes possess common a relatively non-specific and hydrophobic domain. The hydrophobic pentadeca(e)nyl side chain in anacardic acids likely interacts with this hydrophobic domain and disrupts the enzymes' quaternary structure (Tanford, 1980; Hasinoff and Davey, 1989). This concept can be more broadly conceivable. The hydrophobic alkyl side chain should allow for conformational flexibility and interacts with many types of the hydrophobic domain in different enzymes. Anacardic acids' non-specificity of enzyme inhibition supports this assumption. The head and tail structures of anacardic acids suggest that optimization of the lipoxygenase inhibitory activity is possible through a synthetic approach. For example, lipoxygenase inhibitors can be designed by selecting specific head portions that chelate transition metals and by the appropriate lipophilicity of molecules which has a critical impact on the activity. Based on this concept, octyl gallate ( $\mathrm{Ha}$ et al., 2004) and dodecyl gallate (Ha and Kubo, 2007) were found to be potent lipoxygenase inhibitors.

Anacardic acids appear to act as antioxidants in a variety of ways including inhibition of various prooxidant enzymes involved in the production of the reactive oxygen species (Trevisan et al., 2006; Ha and Kubo, 2005; Masuoka and Kubo, 2004) and chelate divalent metal ions (Nagabhushana et al., 1995; Hayashi et al., 2007), but do not quench reactive oxygen species (Kubo et al., 2006). Transition metals are well known as powerful promoters of free radical damage in both the human body (Halliwell and Gutteridge, 1989; Henle and Linn, 1997) and foods (Aruoma and Halliwell, 1991).

In summary, safety is a primary consideration concerning antioxidants in food products. Anacardic acids found in many edible plants, which have been consumed for many years, should be a considerable advantage. Metal chelation capacity of anacardic acids is their additional advantage since it reduces the concentration of the catalyzing transition metal in lipid peroxidation. It is known that chelating agents, which form bonds with a metal, are effective as secondary antioxidants because they reduce the redox potential thereby stabilizing the oxidized form of the metal ion. Hence, anacardic acids were recently suggested to be utilized in 
functional food formulations (Trevisan et al., 2006). Despite the advantage of the isolation from regularly consumed edible plants, biological significance of anacardic acids as lipoxygenase inhibitors in living systems is still largely unknown. Thus, it is not clear if ingested anacardic acids are absorbed into the system through the intestinal tract and delivered to the places where lipoxygenase inhibitors are needed. The relevance of the in vitro experiments in simplified systems compared to in

Arora A., Nair M. G., and Strasburg G. M. (1998), Structure-activity relationships for antioxidant activities of a series of flavonoids an a liposomal system. Free Rad. Biol. Med. 24, 1355-1363.

Aruoma O. I. and Halliwell B. (1991), Free Radicals and Food Additives. Taylor \& Francis, London.

Bhattacharya S. K., Mukhopadhyay M., Rao P. J. R. M., Bagchi A., and Ray, A. B. (1987), Pharmacological investigation on sodium salt and acetyl derivative of anacardic acid. Phytother. Res. 1, 127-131.

Clapp C. H., Banerjee A., and Rotenberg S. A. (1985), Inhibition of soybean lipoxygenase- 1 by $N$-alkylhydroxylamines. Biochemistry 24, 1826-1830.

Coates N. J., Gilpin M. L., Gwynn M. N., Lewis D. E., Milner P. H., Spear S. R., and Tyler J. W. (1994), SB202742, a novel $\beta$-lactamase inhibitor isolated from Spondias mombin. J. Nat. Prod. 57, 654-657.

Copeland R. A. (2000), Enzyme: A Practical Introduction to Structure, Mechanism, and Data Analysis. Wiley-VCH, New York, pp. 266-332.

Cornicelli J. A. and Trivedi B. K. (1999), 15-Lipoxygenase and its inhibition: a novel therapeutic target for vascular disease. Curr. Pharm. Design 5, 11-20.

Gardner H. W. (1991), Recent investigations into the lipoxygenase pathway of plants. Biochim. Biophys. Acta 1084, $221-239$.

Gardner H. W. (1995), Biological roles and biochemistry of the lipoxygenase pathway. HortScience 30, 197205.

Gibian M. J. and Galaway R. A. (1976), Steady-state kinetics lipoxygenase oxygenation of unsaturated fatty acid. Biochemistry 15, 4209-4214.

Grazzini R., Hesk D., Heininger E., Hildenbrandt G., Reddy C. C., Cox-Foster D., Medford J., Craig R., and Mumma R. O. (1991), Inhibition of lipoxygenase and prostaglandin endoperoxide synthase by anacardic acids. Biochem. Biophy. Res. Commun. 176, 775-780.

Grechkin A. (1998), Recent developments in biochemistry of the plant lipoxygenase pathway. Prog. Lipid Res. 37, 317-352.

Ha T. J. and Kubo I. (2005), Lipoxygenase inhibitory activity of anacardic acids. J. Agric. Food Chem. 53, 4350-4354.

Ha T. J. and Kubo I. (2007), Slow-binding inhibition of soybean lipoxygenase-1 by dodecyl gallate. J. Agric. Food Chem. 55, 446-451. vivo experiments for the protection from oxidative damage should be carefully considered. Their further evaluation is needed from not only one aspect, but from a whole and dynamic perspective.

\section{Acknowledgments}

The authors are indebted to Dr. S. Tamura and Ms. I. Hori for performing the enzyme assays at the earlier stage of the work.

Ha T. J., Nihei K. and Kubo I. (2004), Lipoxygenase inhibitory activity of octyl gallate. J. Agric. Food Chem. 52, 3177-3181.

Halliwell B. and Gutteridge J. M. C. (1989), Free Radicals in Biology and Medicine, 2nd ed. Clarendon Press, Oxford.

Hasinoff B. B. and Davey J. P. (1989), The inhibition of a membrane-bound enzyme as a model for anaesthetic action and drug toxicity. Biochem. J. 258, 101-107.

Hayashi A., Taniguchi N., Tsujimoto K., and Kubo I. (2007), Mass spectrometric elucidation of phenolic oxidation process with $\mathrm{Cu}^{2+}$-adducts. J. Mass. Spectrom. Soc. Jpn. 55, 7-13.

Henle E. S. and Linn S. (1997), Formation, prevention, and repair of DNA damage by iron/hydrogen peroxide. J. Biol. Chem. 272, 19095-19098.

Hird N. W. and Milner P. H. (1994), Synthesis and $\beta$-lactamase inhibition of anacardic acids and their analogs. Bioorg. Med. Chem. Lett. 4, 1423-1428.

Irie J., Murata M., and Homma S. (1996), Glycerol-3phosphate dehydrogenase inhibitors, anacardic acids, from Ginkgo biloba. Biosci. Biotechnol. Biochem. 60, $240-243$.

Kemal C., Louis-Flamberg P., Krupinski-Olsen R., and Shorter A. (1987), Reductive inactivation of soybean lipoxygenase- 1 by catechols: a possible mechanism for regulation of lipoxygenase activity. Biochemistry 26, $7064-7072$.

Kris-Etherton P. M. and Keen C. L. (2002), Evidence that the antioxidant flavonoids in tea and cocoa are beneficial for cardiovascular health. Curr. Opin. Lipidol. 13, 41-49.

Kubo I., Komatsu S., and Ochi M. (1986), Molluscicides from the cashew Anacardium occidentale and their large-scale isolation. J. Agric. Food Chem. 34, 970973.

Kubo I., Kim M., Naya K., Komatsu S., Yamagiwa Y., Ohashi K., Sakamoto Y., Hirakawa S., and Kamikawa T. (1987), Prostaglandin synthetase inhibitors from the African medicinal plant Ozoroa mucronata. Chem. Lett. 6, 1101-1104.

Kubo I., Muroi H., Himejima M., Yamagiwa Y., Mera Y., Tokushima K., Ohta S., and Kamikawa T. (1993), Structure and antimicrobial activity relationships of anacardic acids. J. Agric. Food Chem. 41, 1016-1019. 
Kubo I., Kinst-Hori I., and Yokokawa Y. (1994), Tyrosinase inhibitors from Anacardium occidentale fruits. J. Nat. Prod. 57, 545-551.

Kubo J., Lee J. R., and Kubo I. (1999), Anti-Helicobacter pylori agents from the cashew apple. J. Agric. Food Chem. 47, 533-537.

Kubo I., Kinst-Hori I., Chaudhuri S. K., Kubo Y., Sánchez Y., and Ogura T. (2000), Flavonols from Heterotheca inuloides: tyrosinase inhibitory activity and structural criteria. Bioorg. Med. Chem. 8, 1749-1755.

Kubo I., Masuoka N., Ha T. J., and Tsujimoto K. (2006), Antioxidant activity of anacardic acids. Food Chem. 99, $555-562$.

Lodovici M., Guglielmi F., Meoni M., and Dolara P. (2001), Effect of natural phenolic acids on DNA oxidation in vitro. Food Chem. Toxicol. 39, 1205-1210.

Mansuy D., Cucurou C., Biatry B., and Battioni J. P. (1988), Soybean lipoxygenase-catalyzed oxidations by linoleic acid hydroperoxide: different reducing substrates and dehydrogenation of phenidone and BW 755C. Biochem. Biophys. Res. Commun. 151, 339346.

Masuoka N. and Kubo I. (2004), Characterization of xanthine oxidase inhibition by anacardic acids. Biochim. Biophys. Acta 1688, 245-249.

Nagabhushana K. S., Shobha S. V., and Ravindranath B. (1995), Selective ionophoric properties of anacardic acid. J. Nat. Prod. 58, 807-810.

Nelson M. J. (1988), Catecholate complex of ferric soybean lipoxygenase-1. Biochemistry 27, 4273-4278.

Nomura M. and Fujihara Y. (1994), Inhibition of 6$[(8 Z, 11 Z), 14$-pentadecatrienyl]salicylic acid derivatives. J. Jpn. Oil Chem. Soc. 43, 574-578.

Prigge S. T., Boyington J. C., Faig M., Doctor K. S., Gaffney B. J., and Amzel L. M. (1997), Structure and mechanism of lipoxygenases. Biochimie 79, 629-636.
Richard-Forget F., Gauillard F., Hugues M., Jean-Marc T., Boivin P., and Nicolas J. (1995), Inhibition of horse bean and germinated barley lipoxygenases by some phenolic compounds. J. Food Sci. 60, 1325-1329.

Rickert K. W. and Klinman J. P. (1999), Nature of hydrogen transfer in soybean lipoxygenase-1: separation of primary and secondary isotope effects. Biochemistry 38, $12218-12228$.

Ruddat V. C., Whitman S., Holman T. R., and Bernasconi C. F. (2003), Stopped-flow kinetics investigations of the activation of soybean lipoxygenase- 1 and the influence of inhibitors on the allosteric site. Biochemistry 42, 4172-4178.

Sánchez-Ferrer A., Rodríguez-López J. N., García-Cánovas F., and García-Carmona F. (1995), Tyrosinase: A comprehensive review of its mechanism. Biochim. Biophys. Acta 1247, 1-11.

Shobha S. V., Ramadoss C. S., and Ravindranath B. (1994), Inhibition of soybean lipoxygenase-1 by anacardic acids, cardols, and cardanols. J. Nat. Prod. 57, $1755-1757$.

Tanford C. (1980), The Hydrophobic Effect: Formation of Micelles and Biological Membranes, 2nd ed. John Wiley \& Sons, New York.

Toyomizu M., Sugiyama S., Jin R. L., and Nakatsu T. (1993), $\alpha$-Glucosidase and aldose reductase inhibitors: constituents of cashew, Anacardium occidentale, nut shell liquids. Phytother. Res. 7, 252-254.

Trevisan M. T. S., Pfundstein B., Haubner R., Würtele G., Spiegelhalder B., Bartsch H., and Owen R. W. (2006), Characterization of alkyl phenols in cashew (Anacardium occidentale) products and assay of their antioxidant capacity. Food Chem. Toxicol. 44, 188197.

Tyman J. H. P. (1979), Non-isoprenoid long chain phenols. Chem. Soc. Rev. 8, 499-537.

Yamagiwa Y., Ohashi K., Sakamoto Y., Hirakawa S., Kamikawa T., and Kubo I. (1987), Syntheses of anacardic acids and ginkgoic acid. Tetrahedron 43, 3387-3394. 